\title{
Transcriptome sequencing flower petals reveals insights into regulation of flavonoid biosynthesis in Osmanthus fragrans
}

\author{
Y.J. HAN*, M.F. DONG, H.Y. WANG, X.D. WANG, K. LI, and F.D. SHANG* \\ School of Life Sciences, State Key Laboratory of Cotton Biology, State Key Laboratory of Crop Stress \\ Adaptation and Improvement, Key Laboratory of Plant Stress Biology, Laboratory of Plant Germplasm and \\ Genetic Engineering, Henan University, Kaifeng 475004, P.R. China
}

\begin{abstract}
Osmanthus fragrans Lour., one of the top 10 most popular flowers in China, is known for both its beauty and fragrance. It is rich in flavonoids, a class of secondary metabolites with significant neuroprotective, free-radical scavenging, and antioxidant activity. To understand the mechanisms regulating flavonoid biosynthesis, we conducted transcriptome sequencing O. fragrans flowers to analyze gene expressions during the full flowering stage. The RNA was isolated separately from petals of cvs. Yingui and Dangui, which were treated or not with jasmonic acid, salicylic acid, or abscisic acid. A total of 142029 unigenes were denovo assembled, and 50918 unigenes were annotated. The differentially expressed genes were identified, annotated, and classified. The results of transcriptome sequencing and real-time PCR revealed higher expressions of phenylalanine ammonia-lyase (PAL), PAL1, chalcone synthase (CHS), flavanone-3-hydroxylase (F3H), flavonol synthase (FLS), and lower expressions of dihydroflavonol-4-reductase (DFR), anthocyanidin synthase (ANS) in 'Yingui' than in 'Dangui'. Such an expression pattern facilitated the higher accumulation of flavonoids in 'Yingui'. Several genes of the flavonoid biosynthesis pathway were upregulated by jasmonic acid and salicylic acid in both the cultivars leading to flavonoid accumulation in their petals. In the v-myb avian myeloblastosis viral oncogene homolog 1(MYB1)-overexpressing petals, the expressions of PAL, PAL1, CHI, and FLS increased. The results suggest that MYB1 may participate in the flavonoid biosynthesis pathway and regulate the expression of some upstream genes in $O$. fragrans.
\end{abstract}

Additional key words:abscisic acid, DEGs, jasmonic acid, MYB transcription factor, salicylic acid.

\section{Introduction}

Flavonoids are secondary metabolites synthesized by the flavonoid biosynthetic pathway that bestow color to most flowers, fruits, and seeds of plants (Koes et al. 2005). They also play key roles in other plant processes including signaling between plants and microbes (Winkel-Shirley 2001), defense against cold or UV stresses, pathogen attacks, and plant diseases (Debeaujon et al. 2001, Peters and Constabel 2002).

The biosynthetic pathways of flavonoids have been well established, and the respective genes have been mostly isolated (Winkel-Shirley 2001, Tanaka et al. 2008, 2010). Suppression of the anthocyanidin synthase gene in Torenia $\times$ hybrida by RNAi yields white flowers (Nakamura et al. 2006), and suppression of chalcone isomerase gene in transgenic tobacco plants alters flavonoid components and color in both petals and pollen (Nishihara et al. 2005). Suppression of the flavanone-3hydroxylase gene results in a complete loss of the original orange/reddish color and emission of methyl benzoate in carnations (Zuker et al. 2002). A higher expression of the flavone synthase (FNS) gene and/or suppression of the dihydroflavonol-4-reductase (DFR) gene may be necessary to achieve a higher flavone/anthocyanin ratio and obtain a bluer petunia by co-pigmentation (Tsuda et al. 2004). Expression of the gerbera DFR gene and suppressions of two endogenous genes flavonol synthase $(F L S)$ and flavonoid-3'-hydroxylase $\left(F 3^{\prime} H\right)$ increase the accumulation of pelargonidin pigments in tobacco flowers (Nakatsuka et al. 2007). Retransformation of a transgenic plant expressing gerbera $D F R$ and suppression of flavonoid-3',5'-hydroxylase (F3' $\left.5^{\prime} \mathrm{H}\right)$ activity results in

Submitted 12 December 2018, last revision 28 October 2019, accepted 28 November 2019.

Abbreviations: ANS - anthocyanidin synthase; CHI - chalcone isomerase; CHS - chalcone synthase; DEGs - differentially expressed genes; DFR - dihydroflavonol 4-reductase; F3H - flavanone-3-hydroxylase; F3'H - flavonoid-3'-hydroxylase; FLS - flavonol synthase; MYB - v-myb avian myeloblastosis viral oncogene homolog; PAL - phenylalanine ammonia-lyase; PAL1- phenylalanine ammonialyase 1; R2R3 MYB - two repeats MYB domains.

Acknowledgements: This research was supported by the National Natural Science Funds in China (Nos U1604114 and 31400521 ).

*Corresponding author; fax: (+86) 371 23887799, e-mail:wolianghan@163.com_and fudeshang@henu.edu.cn 
accumulation of predominantly pelargonidin derivatives in Osteospermum hybrida flowers (Seitzet al. 2007).

The regulation of the flavonoid pathway has been studied extensively in several plants (Broun 2005, Koes et al. 2005, Ramsay and Glover 2005). The two repeats of MYB domain (R2R3 MYB) transcription factors regulate gene expression by binding to the regulatory elements in the promoters of structural genes related to flavonoid biosynthesis (Koes et al. 2005). Expressions of two maize R2R3 MYB transcription factor genes leaf color $(L C)$ and colorless $1(\mathrm{Cl})$ in tomato are sufficient to upregulate the flavonoid pathway in fruit flesh (Bovy et al. 2002). Overexpression of a tomato MYB transcription factor gene activation tagged insertion lines 1 (ANT1) upregulates genes encoding proteins in the anthocyanidin biosynthesis, which results in purple spotting on fruit epidermis and pericarp (Mathews et al. 2003). It has been proved that MYB transcription factors participate in the regulation of the flavonoid pathway in maize (Hernandez et al. 2004), snapdragon (Schwinn et al. 2006), petunia (Spelt et al. 2000, Quattrocchio et al. 2006), Ipomoea (Morita et al. 2006), tomato (Mathews et al. 2003), apple (Takos et al. 2006, Espley et al. 2007, Vimolmangkang et al. 2013), and Arabidopsis (Quattrocchio et al. 1999, Nesi et al. 2001, Baudry et al. 2010, Gonzalez et al. 2010, Mondal et al. 2017).

Osmanthus fragrans, also known as sweet osmanthus, is one of the top 10 domesticated flowers and a popular landscaping plant. It is valued for its beauty and fragrance and has been cultivated for over 2500 years in China. Its numerous cultivars are classified into four cultivar groups Yingui, Jingui, Dangui, and Sijigui based on different flowering seasons, flower colors and inflorescence types (Xiang and Liu 2007, He et al. 2017). In the present study, we performed transcriptome sequencing for sweet osmanthus petals using the Illumina sequencing platform. The aim of this work was to determine if differences in the gene expressions of the flavonoid biosynthesis pathway is responsible for the different content of flavonoids in petals of 'Yingui' and 'Dangui'. The results could advance our understanding of flavonoid metabolism and contribute to utilization of flavonoid compounds and cultivation of new cultivars of sweet osmanthus.

\section{Materials and methods}

Plants and cultivation: Freshly cut flowering branches of Osmanthus fragrans Lour. (cv. 'Baijie' from the Yingui group and cv. 'Chenghong Dangui' from the Dangui group) were incubated under a $12-\mathrm{h}$ photoperiod, an irradiance of $80 \mu \mathrm{mol} \mathrm{m} \mathrm{m}^{-2} \mathrm{~s}^{-1}$, a constant temperature of $22{ }^{\circ} \mathrm{C}$, and a relative humidity of $70 \%$ (Han et al. 2016, 2019). The flowering branches of both cultivars 'Chenghong Dangui' (D) and 'Baijie' (Y) were divided into four groups. One group of branches was not treated (D0 and Y0) and the three remaining groups were treated with $50 \mu \mathrm{M}$ jasmonic acid (JA; DJ and YJ), $5 \mathrm{mM}$ salicylic acid (SA; DS and YS), or $20 \mu \mathrm{M}$ abscisic acid (ABA; DA and YA) for $3 \mathrm{~h}$. The flowers at full flowering stage were collected and frozen in liquid nitrogen and stored at $-80{ }^{\circ} \mathrm{C}$ until use.

Extraction of RNA: Total RNA was isolated from petals using a plant RNA kit (Transgen Biotech, Beijing, China). The RNA concentration was determined by a spectrophotometer (Nanodrop Technologies, Wilmington, DE, USA), and RNA integrity was evaluated by an Agilent 2100 bioanalyzer (Agilent Technologies, Santa Clara, CA, USA) (more detail in Han et al. 2014b).

Library construction and transcriptome sequencing: A total amount of $3 \mu \mathrm{g}$ of RNA per sample was used. Sequencing libraries were generated using an $N E B N e x t \AA$ Ultra $^{\mathrm{TM}}$ RNA library preparation kit for Illumina ${ }^{\circledR}$ (NEB, Ipswich, USA) following the manufacturer's recommendations, and index codes were added to attribute sequences to each sample. The mRNA was isolated from petals and purified using poly-T oligo-attached magnetic beads. The first cDNA strand was produced using random hexamer primers and reverse transcription kits. The second cDNA strand was synthesized by DNA polymerase I and RNase H. Remaining overhangs were converted into blunt ends via exonuclease/polymerase activities. After adenylation of 3 ' ends of DNA fragments, $N E B$ Next adapter oligonucleotides with a hairpin loop structure were ligated to prepare for hybridization. In order to select cDNA fragments of preferentially 150-200 bp in length, the library fragments were purified with an AMPure XP system (Beckman Coulter, Beverly, USA). Then, $3 \mathrm{~mm}^{3}$ of the uracil-specific excision reagent (USER) enzyme $(N E B)$ was used with size-selected, adaptor-ligated cDNA at $37^{\circ} \mathrm{C}$ for $15 \mathrm{~min}$ followed by $5 \mathrm{~min}$ at $95{ }^{\circ} \mathrm{C}$ before PCR. Then, PCR was performed with phusion high-fidelity DNA polymerase, universal PCR primers, and an index primer. At last, PCR products were purified (the AMPure $X P$ system) and library quality was assessed on an Agilent Bioanalyzer 2100 system. Clustering index-coded samples was performed on a cBot Cluster Generation system using a TruSeq PE Clusterkit v3-cBot-HS (Illumina) according to the manufacturer's instructions. After cluster generation, the library preparations were sequenced on an Illumina platform and paired-end reads were generated.

Sequence data assembly and functional annotation: To obtain high quality data for sequence assembly and analysis, raw data (raw reads) in fastq format were firstly processed through in-house perl scripts. In this step, clean data (clean reads) were obtained by removing reads containing an adapter, reads containing ploy-N and low quality reads with $\mathrm{Q}<20$ from raw data using the TRINITY (Grabherr et al. 2011) RNASEQ_ROOT/util/normalize by_kmer_coverage. The pl. transcriptome assembly was accomplished using TRINITY with a 'min_kmer_cov' set to 2 by default and all other parameters set default.

Unigene sequences were subjected to the Blastx algorithm of $\mathrm{Nr}$ (NCBI non-redundant protein sequences), $N t$ (NCBI non-redundant nucleotide sequences), Swiss-Prot (a manually annotated and reviewed protein sequence database) (Bairoch and Apweiler 1999), COG/KOG 
(clusters of orthologous groups of proteins) (Sonnhammer and Koonin 2003, Tatusovet al. 2003), and KEGG ortholog database (Kanehisa et al. 2004) to obtain a protein with the highest similarity. Thus, we obtained the annotation information of protein function. The $K E G G$ database was used to assign unigenes to pathways.

Unigene differential expression analysis: Differential expression analysis was performed using the DESeq2R package (Love et al. 2014) for determining differential expression in digital gene expression data using a model based on the negative binomial distribution. The resulting $P$ values were adjusted using the Benjamini and Hochberg's approach for controling the false discovery rate. Genes with an adjusted $P$-value $<0.01$ found by $D E S e q 2$ were assigned as differentially expressed. The expressions of unigenes were calculated using the fragments per kilobase million (FPKM) method. The expressions of differentially expressed genes (DEGs) were computed by the following formula: FPKM = cDNA fragments/[mapped fragments (millions) $\times$ transcript length $(\mathrm{kb})]$. The FPKM results can be directly used to compare gene expression within differential samples.

Isolation and sequence analysis of $O f M Y B 1$ : For isolation of $O f M Y B 1$ from $O$. fragrans, two primers MYB1f and MYB1r (Table 1 Suppl.) were synthesized to amplify the full length $M Y B 1$ gene. A PCR amplification was performed with the primers. After an initial $94{ }^{\circ} \mathrm{C}$ 4 min denaturation step, 35 cycles were run, each with $45 \mathrm{~s}$ of denaturation at $94{ }^{\circ} \mathrm{C}$, followed by $45 \mathrm{~s}$ annealing at $56^{\circ} \mathrm{C}$, and $60 \mathrm{~s}$ extension at $72{ }^{\circ} \mathrm{C}$. The PCR product was cloned into the pMD19-T vector (TaKaRa, Dalian China) and sequenced. We then compared the amino acid sequence of OfMYB1 with MYBs from other plant species. These sequences were aligned using Clustal $W$ v. 1.83. Phylogenetic analysis was performed using MEGA 4.1. A neighbor joining tree was constructed according to the distance matrix that had been computed.

Real-time quantitative PCR analysis was utilized to evaluate the relative expressions of related genes. Gene specific primers were designed using the Primer 5.0 software (Primer Biosoft International, Palo Alto, CA, USA) and listed in Table 1 Supp 1. Each $20 \mathrm{~mm}^{3}$ of reaction included $10 \mathrm{~mm}^{3}$ of SYBR Green $q R T-P C R$ mix. The following amplification program was used: the initial denaturation at $95^{\circ} \mathrm{C}$ for 3 min followed by 40 cycles of denaturation at $95^{\circ} \mathrm{C}$ for $7 \mathrm{~s}$, annealing at $60^{\circ} \mathrm{C}$ for $10 \mathrm{~s}$, and extension at $72{ }^{\circ} \mathrm{C}$ for $50 \mathrm{~s}$. The sweet osmanthus $\beta$-actin gene was used as an internal reference, and zelative expressions were computed by the $2^{-\Delta \Delta \mathrm{Ct}}$ method (Livak and Schmittgen 2001). Each sample was prepared in three to five biological replicates. The SPSS v. 12.0 (SPSS Inc., Chicago, IL, USA) software was used for statistical analysis.

Transient overexpression of OfMYB1 in $O$. fragrans flower petals: Transient overexpression of OfMYB1 (accession No. KM504383) in petals of $O$. fragrans
'Dangui' in the full flowering stage was performed according to Han et al. (2016). The MYB1 coding region was amplified using gene specific primers (MYB1f1 and MYB1r1) (Table 1 Suppl.) and subcloned into the pHBT vector. The resulting HBT-OfMYB1 vector (35Spro:MYB1-GFP) and HBT vector (35Spro:GFP, control) were transformed into Agrobacterium tumefaciens strain EHA105 using the freeze-thaw method. After overnight culture, the Agrobacterium was resuspended in $10 \mathrm{mM} \mathrm{MgCl}_{2}, 10 \mathrm{mM} \mathrm{MES}, 150 \mathrm{mM}$ acetosyringone until reaching an absorbance of 0.6 at $600 \mathrm{~nm}$. The petals were cut into a $2 \mathrm{~mm}$ size, vacuum infiltrated with $A$. tumefaciens for $15 \mathrm{~min}$, and kept in the dark in $5 \%(\mathrm{~m} / \mathrm{v})$ sucrose for $36-48 \mathrm{~h}$. The RNA extracted from infiltrated petals was analyzed using real-time quantitative PCR (qPCR) (Han et al. 2016).

\section{Results}

After filtering the raw sequences, 27475198 (in D0), 24547401 (in DJ), 26079942 (in DS), 27722398 (in DA), 28420554 (in Y0), 31683095 (in YJ), 34016142 (in YS), and 27722398 (in YA) high quality reads were obtained (Table 2 Suppl.). Using the TRINITY assembly software, 142029 unigenes were assembled with an $\mathrm{N}_{50}$ length of $1300 \mathrm{bp}$. The length distributions of contigs, transcripts, and unigenesare shown in Table 3 Suppl. Transcriptome sequencing data have been deposited in the NCBI sequence read archive (SRA) database with accession number PRJNA565564.

The highest number of upregulated (2 223) and downregulated (2 171) unigenes was observed between Y0 and D0. The lowest number of upregulated (896) unigenes was observed between D0 and DJ, and the lowest number of downregulated (638) unigenes was observed between Y0 and YA (Table 4 Suppl.). There were 494 overlap DEGs between $\mathrm{Y} 0$ and $\mathrm{YJ}, \mathrm{Y} 0$ and $\mathrm{YS}, \mathrm{Y} 0$ and $\mathrm{YA}$ and $\mathrm{Y} 0$ and D0 combinations, and 684 overlap DEGs between D0 and DJ, D0 and DS, D0 and DA and Y0a and D0 combinations (Fig. 1 Suppl.). The results suggest that the expression of 494 and 684 genes were influenced by the three plant hormones (JA, SA, and ABA) in 'Yingui' and 'Dangui', respectively.

A total of 50918 unigenes were annotated by sequencebased alignments in $C O G / K O G$ (Sonnhammer and Koonin 2003, Tatusov et al. 2003), GO (Harris et al. 2004), KEGG (Kanehisa et al. 2004), Pfam (Finn et al. 2007), Swiss-Prot (Bairoch and Apweiler 1999), NCBI, and NR databases (https://www.ncbi.nlm.nih.gov/) (Table 5 Suppl.) using the $B L A S T X$ algorithm (Altschul et al. 1990) with an E-value less than $1 \mathrm{e}^{-5}$ demonstrating a high sequence homology.

Unigene sequences were subjected to the BLASTX algorithm of $\mathrm{Nr}$, Nt, Swiss-Prot, COG/KOG, and the $K E G G$ ortholog database to obtain a protein with the highest similarity; we obtained the annotation information of protein function. The $K E G G$ database was used to assign unigenes to pathways.

Of the 50918 unigenes, 26941 unigenes were annotated in the GO database, and their functions were 
Table 1. The number of annotated unigenes.

\begin{tabular}{lllllllll}
\hline DEG Set & Annotated & COG & GO & KEGG & KOG & Pfam & Swiss-Prot & Nr \\
\hline D0_vs_DJ & 1448 & 463 & 822 & 436 & 682 & 1126 & 1096 & 1439 \\
D0_vs_DS & 1962 & 610 & 1096 & 571 & 968 & 1502 & 1488 & 1951 \\
D0_vs_DA & 2184 & 651 & 1205 & 633 & 1000 & 1681 & 1647 & 2173 \\
Y0_vs_D0 & 3305 & 1027 & 1851 & 1100 & 1790 & 2394 & 2336 & 3272 \\
Y0_vs_YJ & 1689 & 563 & 1013 & 537 & 852 & 1309 & 1287 & 1681 \\
Y0_vs_YS & 1727 & 565 & 984 & 558 & 893 & 1342 & 1308 & 1722 \\
Y0_vs_YA & 1538 & 490 & 873 & 484 & 725 & 1188 & 1154 & 1528 \\
\hline
\end{tabular}

Table 2. Identified flavonoid synthesis pathway related unigenes.

\begin{tabular}{|c|c|c|c|c|c|c|}
\hline \multirow[t]{2}{*}{ Unigene ID } & \multicolumn{2}{|c|}{ 'Dangui' vs.'Yingui' } & \multirow{2}{*}{$\begin{array}{l}\text { Nucleotide } \\
\text { length }\end{array}$} & \multirow{2}{*}{$\begin{array}{l}\text { Protein } \\
\text { length }\end{array}$} & \multirow[t]{2}{*}{ Nr_annotation } & \multirow[t]{2}{*}{ Accession number } \\
\hline & $\log 2 \mathrm{FC}$ & regulated & & & & \\
\hline BMK.70848 & 0.20407 & up & $2136 \mathrm{bp}$ & 712 aа & $\begin{array}{l}\text { phenylalanine ammonia-lyase [Olea } \\
\text { europaea }\end{array}$ & MN512442 \\
\hline CL35673 & 2.10227 & up & $2139 \mathrm{bp}$ & 713 aа & $\begin{array}{l}\text { phenylalanine ammonia-lyase } \\
1[\text { Nelumbo nucifera }]\end{array}$ & MN512443 \\
\hline CL859 & 5.17495 & up & 1170 bp & 390 aа & chalcone synthase [Olea europaea] & KR604813 \\
\hline CL35874 & 0.58434 & up & $747 \mathrm{bp}$ & 249 aа & chalcone isomerase [Olea europaea] & ALL27265 \\
\hline CL36577 & 4.23624 & up & $1101 \mathrm{bp}$ & 367 aа & $\begin{array}{l}\text { flavanone 3-hydroxylase } \\
\text { [Gossypium hirsutum] }\end{array}$ & MN512444 \\
\hline CL40977 & 5.71978 & up & $1002 \mathrm{bp}$ & 334 aа & $\begin{array}{l}\text { Flavonol synthase }[\text { Petunia } x \\
\text { hybrida }]\end{array}$ & MN512445 \\
\hline BMK.70048 & -1.704667 & down & 1128 bp & 376 aа & $\begin{array}{l}\text { dihydroflavonol reductase } \\
{[\text { Forsythia } x \text { intermedia }]}\end{array}$ & ANA96271 \\
\hline BMK.46512 & -2.670299 & down & 1146 bp & 382 aа & $\begin{array}{l}\text { anthocyanidin synthase }[\text { Forsythia } \times \\
\text { intermedia }]\end{array}$ & MN512446 \\
\hline CL35948 & 0.15411 & up & 585 bp & 195 aа & $\begin{array}{l}\text { Myb-related protein } 305 \\
\text { (Antirrhinum majus) }\end{array}$ & KM504383 \\
\hline
\end{tabular}

divided into biological process, cellular component, and molecular function (Fig. 2 Suppl.). The largest number of annotated genes in the three functions were detected in the $\mathrm{Y} 0-v s-\mathrm{D} 0$ combination: 'biological process' (GO:0008150) comprised 20848 unigenes (40.94\%), 'cellular_component' (GO:0005575) included 13325 unigenes (26.17\%), and 'molecular function' (GO:0003674) consisted of 22357 unigenes (43.91\%). Among the seven DEG sets, the Y0-vs-D0 combination had the largest number of annotated genes (3 305), and 1027 DEGs were aligned to the $C O G$ database (Table 1).

The unigenes annotated to the $C O G$ database were classified into 25 different functional classes in Y0-vs-D0 (Fig. 3 Suppl.). The cluster of general function prediction $(276 ; 18.02 \%)$ presented the largest group followed by replication, recombination, and repair $(160 ; 10.44 \%)$, transcription $(134 ; 11.9 \%)$, signal transduction mechanisms $(125 ; 12.28 \%)$, carbohydrate transport and metabolism $(116 ; 7.57 \%)$, posttranslational modification, protein turnover, chaperones $(102 ; 6.66 \%)$, and amino acid transport and metabolism $(102 ; 6.66 \%)$.

A total of 16985 unigenes were annotated to the $K E G G$ database, and 1091 DEGs were assigned to 117 different
$K E G G$ pathways. The $K E G G$ categories were mainly classified into five groups: cellular process, environmental information processing, genetic information processing, metabolism, and organismal systems. The greatest number of DEGs were in starch and sucrose metabolisms (ko03010, 44 DEGs, 3.14\%) followed by biosynthesis of amino acids (39), plant hormone signal transduction (38), protein processing in endoplasmic reticulum (37), carbon metabolism (33), and phenylpropanoid biosynthesis (26) (Fig. 4 Suppl.).

Flavonoid accumulation can be regulated by the expressions of flavonoid biosynthesis genes. Using the analysis platform (http://www.biomarker.com.cn/ biocloud), eight DEGs were selected for further analysis. These DEGs were the key genes related to flavonoid biosynthesis. Of those, six DEGs were upregulated: BMK.70848 (PAL), CL35673 (PAL1), CL859 (CHS), CL35874 (CHI), CL36577 (F3H), and CL40977 (FLS), and two DEGs were downregulated: BMK.70048 (DFR), and BMK.46512 (ANS) in 'Yingui' (Table 2, Figs. 1, 2). The results of the gene expression profiles of other DEG sets exhibited small differences in transcriptions (Fig. 1).

Sequence analysis of OfMYB1 shows that a unigene 


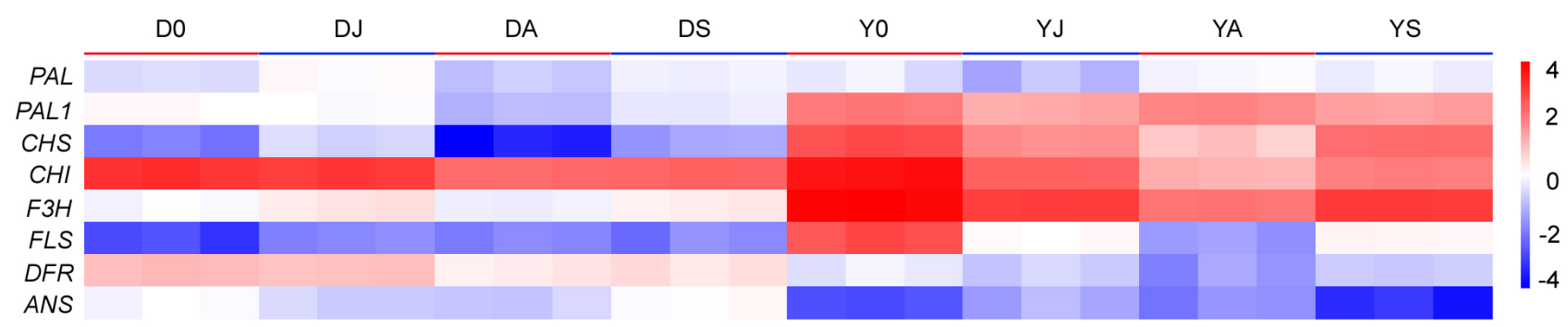

Fig. 1. A heat-map depicting normalized log2-fold changes in mRNA expression inferred from RNAseq data for transcripts involved in flavonoid biosynthesis. DO, DJ, DA, DS, YO, YJ, YA, and YS, please refer to Materials and methods section. PAL - phenylalanine ammonia-lyase, PAL1 - phenylalanine ammonia-lyase 1, CHS -chalcone synthase, $\mathrm{CH}$-chalcone isomerase, F3H -flavanone-3hydroxylase, FLS -flavonol synthase, DFR - dihydroflavonol-4-reductase, ANS -anthocyanidin synthase.

CL35948 was slightly upregulated in 'Yingui'. Sequence comparison indicates a high similarity between this unigene and the one encoding Antirrhinum majus mybrelated protein 305 . The unigene appeared to be a full length sequence of 585 bp encoding a polypeptide of 195 amino acid residues and named O. fragrans MYB1 (OfMYB1). Sequence alignment reveals that MYB1 shared a high amino acid sequence identity with Antirrhinum majus myb305 (AmMYB305), Coffea eugenioides MYB305, Sesamum indicum MYB305, Gerbera hybrid cultivar MYB305, Petunia $\times$ hybrida EOBI, Mucuna pruriens MYB305, Vitis vinifera MYB24, and Capsicum baccatum MYB21 (Fig. 5 Suppl.). A phylogenetic tree was constructed based on the amino acids of OfMYB1 and some MYBs from other plant species. The OfMYB1 shows a high similarity with Antirrhinum majus MYB305, Coffea eugenioides MYB305, and Sesamum indicum MYB305 (Fig. 5 Suppl.).

To investigate whether the results of gene expression profiles obtained from RNA-seq were accurate, the relative expressions of the eight genes related to the flavonoid biosynthesis pathway were examined by real-time qPCR (Fig. 3). Compared with 'Dangui', the transcriptions of $P A L 1, C H S, F 3 H$, and FLS in petals of 'Yingui' increased

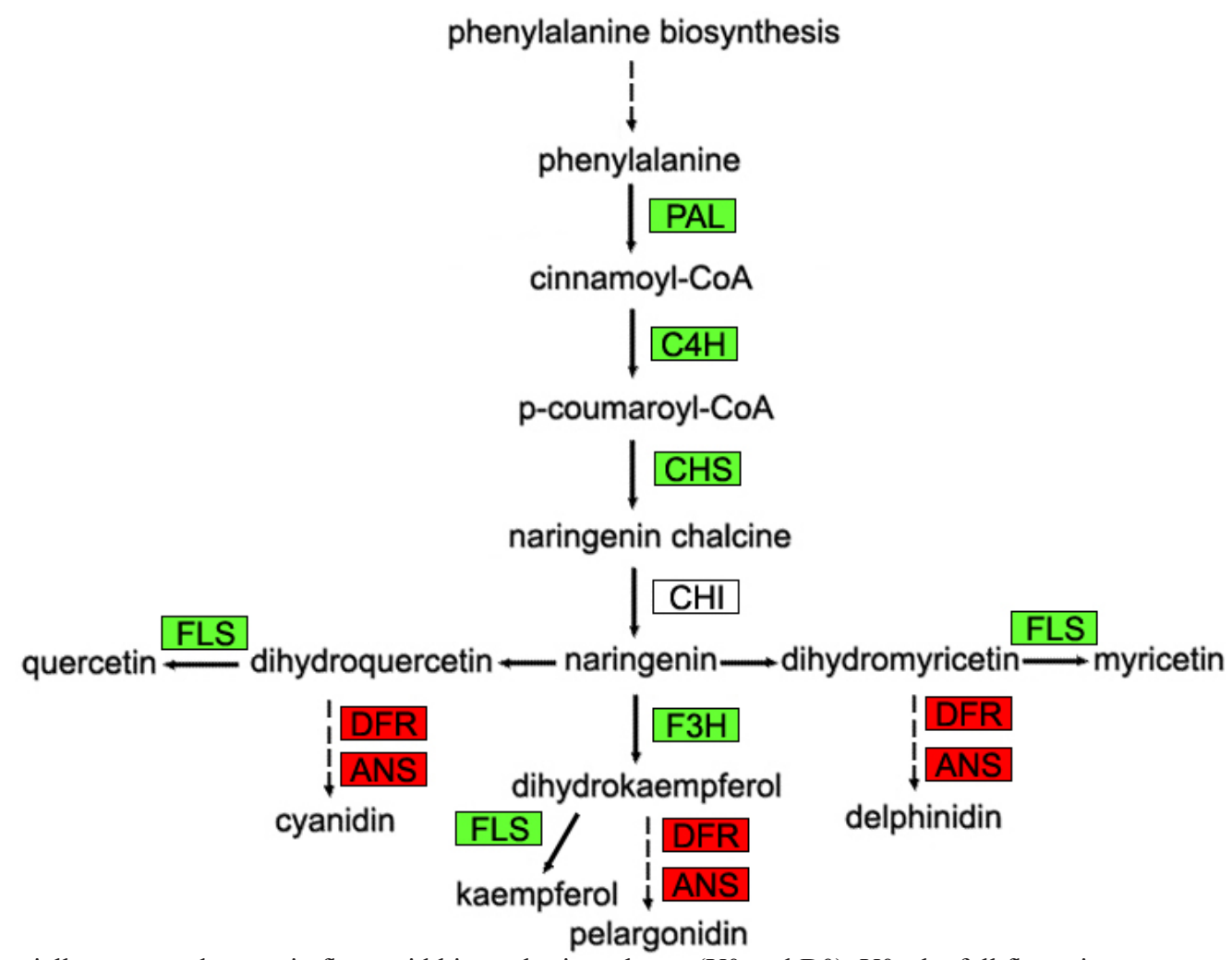

Fig. 2. Differentially expressed genes in flavonoid biosynthesis pathway ( $Y 0$ and D0). Y0 -the full flowering stage petals of 'Yingui'; D0 -the full flowering stage petals of 'Dangui'. Red represents upregulated genes and green represents downregulated genes. PAL phenylalanine ammonia-lyase, $\mathrm{C} 4 \mathrm{H}$-cinnamate-4-hydroxylase, CHS -chalcone synthase, $\mathrm{CHI}$-chalcone isomerase, $\mathrm{F} 3 \mathrm{H}$-flavanone-3hydroxylase, FLS -flavonol synthase, DFR -dihydroflavonol-4-reductase, ANS -anthocyanidin synthase. 

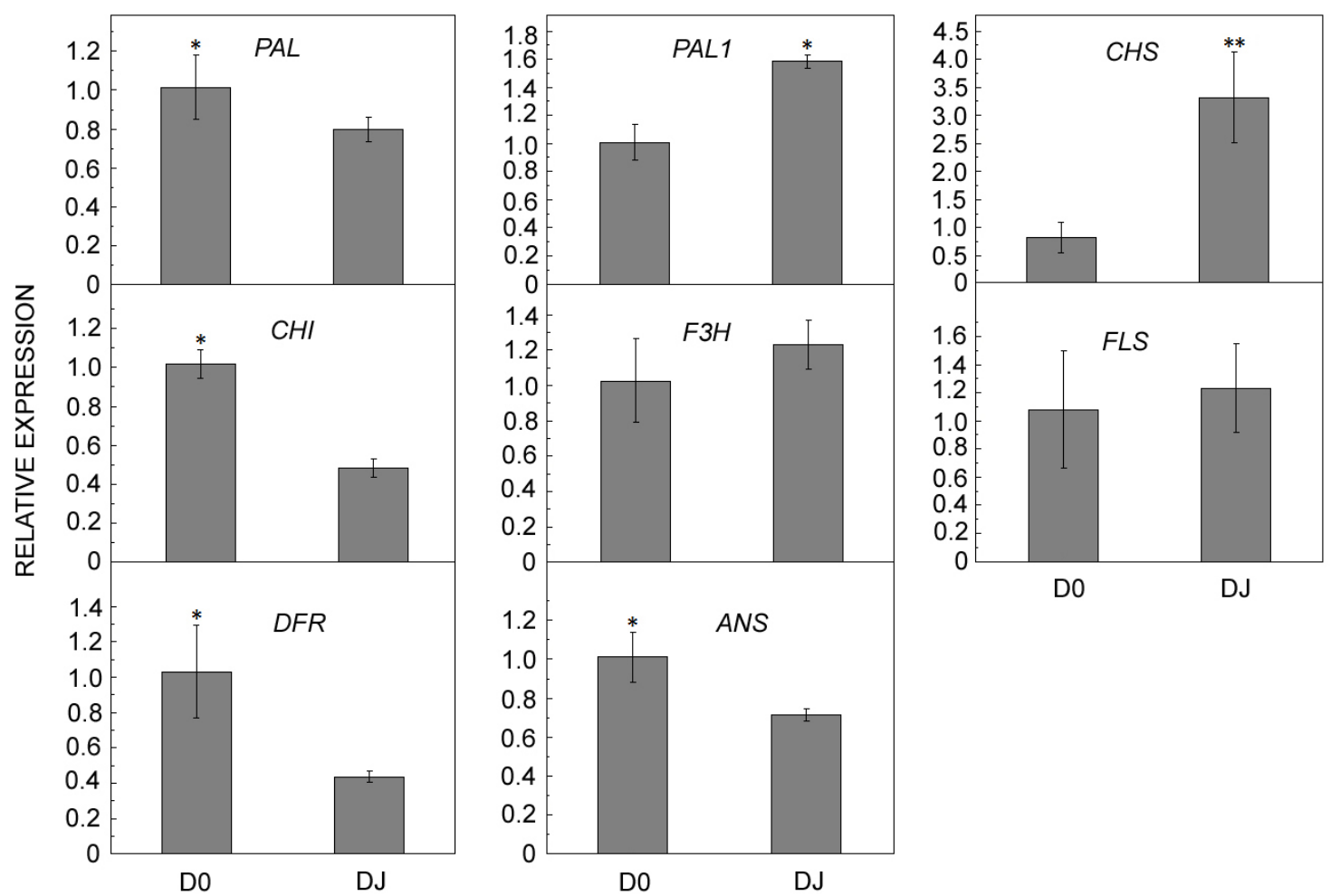

Fig. 3. Transcriptions of genes involved in flavonoid biosynthesis pathway in $\mathrm{Y} 0$ and D0. Y0 -the full flowering stage petals of 'Yingui', $\mathrm{D} 0$-the full flowering stage petals of 'Dangui'. For abbreviations see Fig. 2. Means \pm SDs, $n=3$. Statistically significant differences at $*-P<0.05$ and $* *-P<0.01$.
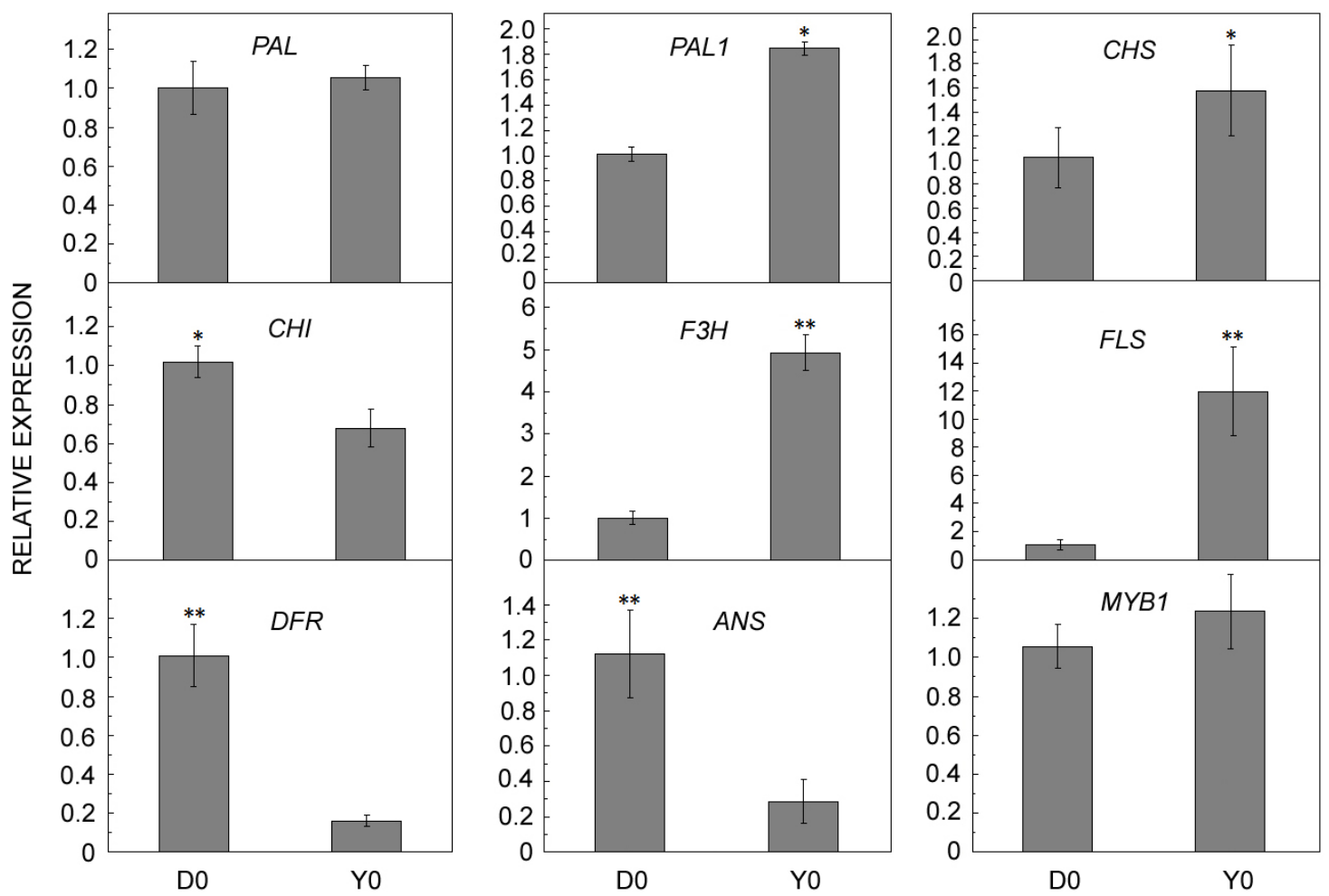

Fig. 4. Transcriptions of genes involved in flavonoid biosynthesis pathway in D0 and DJ. D0 -the full flowering stage petals of 'Dangui', DJ -the full flowering stage petals of 'Dangui' treated with JA. For abbreviations see Fig. 2. Means \pm SDs, $n=3$. Statistically significant differences at $*-P<0.05$ and $* *-P<0.01$. 

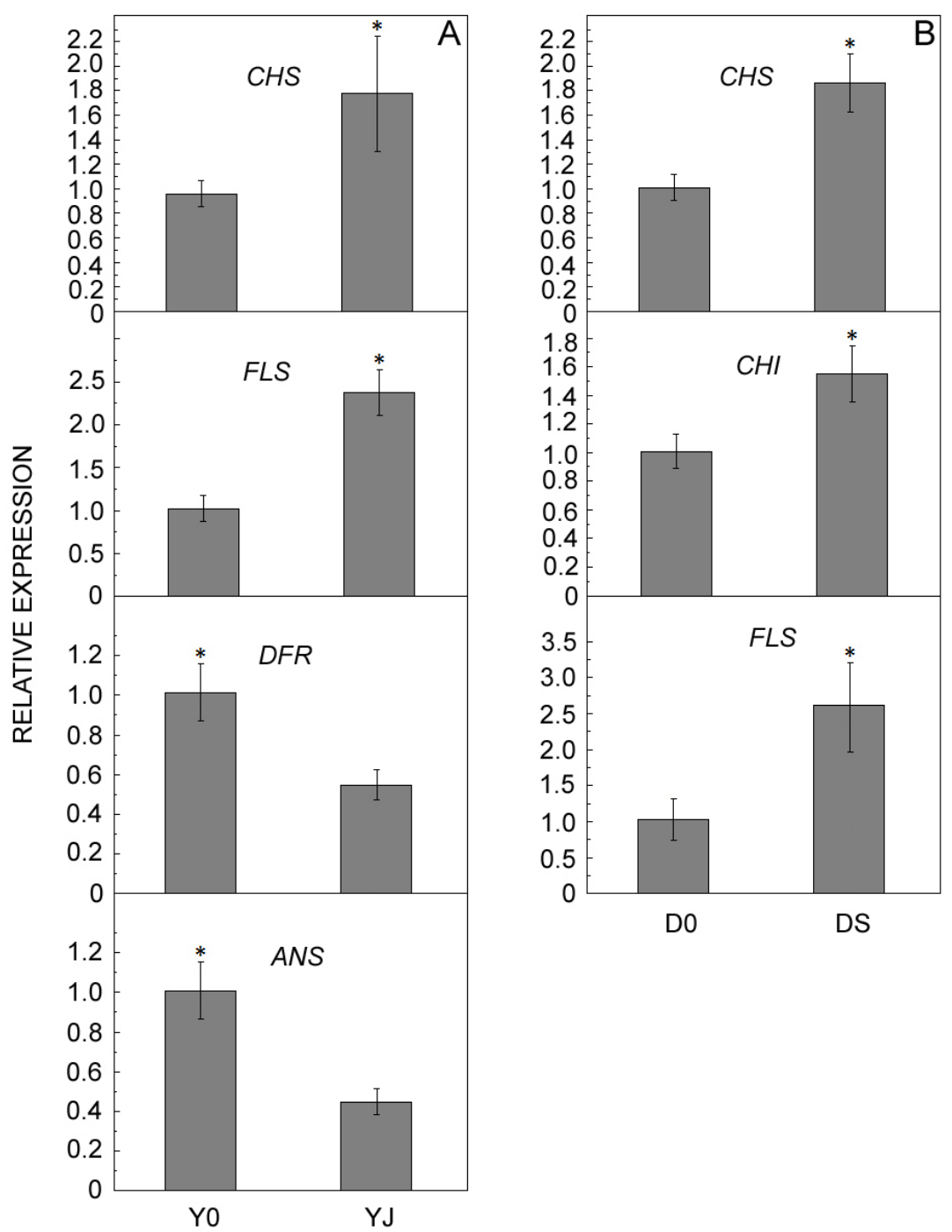

Fig. 5. Transcriptions of some genes involvedin flavonoid biosynthesis pathwayin Y0 and YJ $(A)$ and D0 and DS $(B)$. Y0 -the full flowering stage petals of 'Yingui' , YJ -the full flowering stage petals of 'Yingui' treated with JA; D0 -the full flowering stage petals of 'Dangui',DS -the full flowering stage petals of 'Dangui' treated with SA. For abbreviations see Fig. 2. Means \pm SDs, $n=3$. Statistically significant differences at $*$ - $P<0.05$.

0.8-, 0.5-, 3.9-, and 10.9-fold, respectively, and those of $C H I, D F R$, and ANS were reduced 0.4-, 5.3-, and 2.9fold, respectively. Moreover, there was no significant difference in the expression of $P A L$ between 'Yingui' and 'Dangui' (Fig. 3). Thus, the qPCR analysis corroborated the expression patterns of the eight genes inferred by RNA-seq. Both RNA-seq and qPCR analysis show that the expression of OfMYB1 in 'Yingui' petals was slightly higher than in 'Dangui' (Table 2, Fig. 3).

To determine the effects of hormones on the expressions of these genes, the flowers treated with JA, SA, and ABA for $3 \mathrm{~h}$ were examined by real-time qPCR. According to the RNA-seq results, PAL1, CHS, F3H, and FLS were upregulated, and PAL1,CHI, DFR, and ANS were slightly downregulated after the treatment with JA (Fig. 1). In DJ, the transcriptions of PAL1, CHS, F3H, and FLS increased 0.58-, 2.43-, 0.21-, and 0.13-fold, and those of PAL, CHI, $D F R$, and $A N S$ were reduced 0.2-, 0.52-, 0.58-, and 0.3- fold, respectively, when compared with those in D0 and as indicated by qPCR (Fig. 4). The transcriptions of the genes obtained by RNA-seq were consistent with those detected by the qPCR. As indicated by the qPCR results, $C H S$ and FLS increased 0.81- and 1.35-fold, and DFR and $A N S$ were reduced 0.45 - and 0.55 -fold in YJ, respectively,

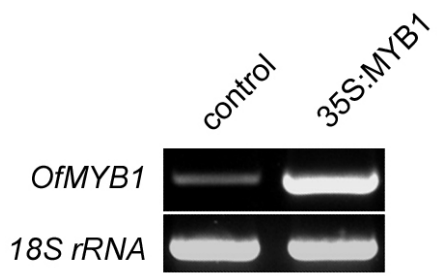

Fig. 6. Detection of $v$-myb avian myeloblastosis viral oncogene homolog 1 (MYB1) transcripts in control and transiently transformed petals using reverse transcription polymerase chain reaction detection. 

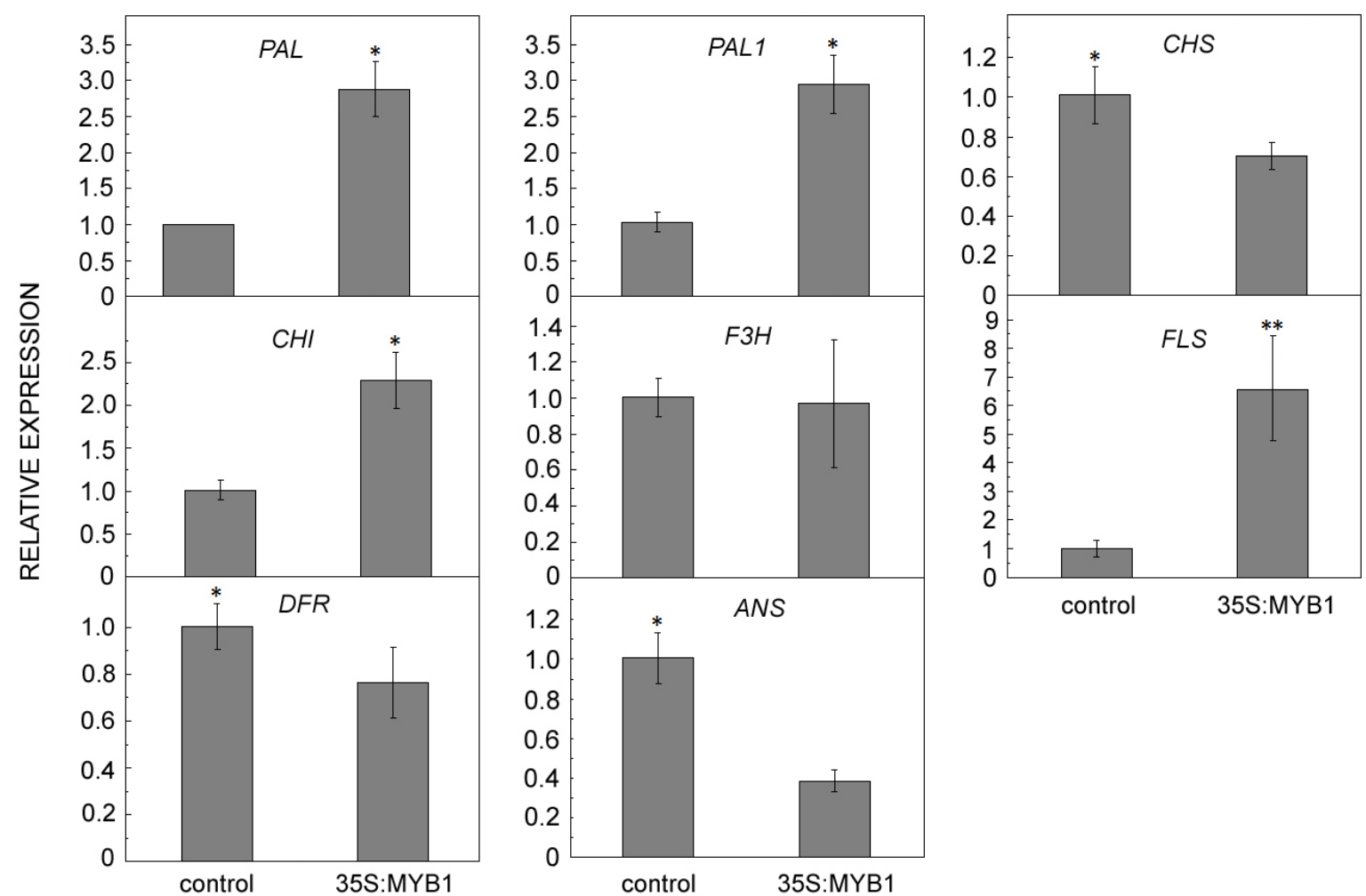

Fig. 7. Comparison of flavonoid biosynthesis pathway gene transcriptions in wild type 'Dangui' flower petals (control) and transiently transformed petals (35S:MYB1). For abbreviations see Fig. 2. Means \pm SDs, $n=3$. Statistically significant differences at $*-P<0.05$ and $* *-P<0.01$.

when compared with their expressions in Y0 (Fig. 5A). The transcriptions of $P A L, P A L 1$, and $C H I$ were not significantly different between Y0 and YJ. These results suggest that the upstream genes of the flavonoid biosynthesis pathway were upregulated and the downstream genes were downregulated promoting the accumulation of flavonoids in sweet osmanthus flowers treated with JA.

In flowers treated with $\mathrm{SA}$, the transcriptions of CHS, CHI, and FLS increased 0.85-, 0.56-, and 1.58-fold, respectively, as indicated by qPCR (Fig. $5 B$ ). The transcriptions of the remaining genes were not significantly different between D0 and DS. These results suggest that the treatment of flowers with SA upregulated the transcription of some upstream genes of the flavonoid biosynthesis pathway, resulting in an increased flavonoid content. There were no significant changes in the expressions of the genes between the flowers before and after the treatment with ABA implying that ABA did not alter the flavonoid metabolism in flowers.

To determine the ability of OfMYB1 to upregulate the transcriptions of the flavonoid biosynthesis-related genes, OfMYB1 was transiently transformed into 'Dangui' flower petals. The analyses of flowers transformed with 35S:OfMYB1 and 35S:GFP revealed an overexpression of $O F M Y B 1$ in the former (Fig. 6). The transcriptions of $P A L, P A L 1, C H I$, and FLS were affected by the OfMYB1 overexpression: their expressions increased 1.8-, 1.9-, 1.3-, and 5.5-fold, respectively in OfMYB1-overexpressing petals compared with the control (Fig. 7).

\section{Discussion}

Transcriptome sequencing represents an attractive alternative to whole-genome sequencing because it analyzes only transcribed portions of the genomeand avoiding non-coding and repetitive sequences (Margulies et al. 2005, Huse et al. 2007, Novaes et al. 2008). The transcriptome provides information on gene expression, gene regulation, and amino acid content of proteins. Therefore, transcriptome analysis is essential to interpreting the functional elements of the genome and revealing the molecular constituents of cells and tissues (Wang et al. 2009, Wei et al. 2011). In this study, using the TRINITY de novo assembly program, short-read sequences were assembled into 128098 unigenes with an N50 length of $882 \mathrm{bp}$ and with a mean length of $599 \mathrm{bp}$. It was annotated 70915 unigenes. The DEGs were identified, annotated, and classified. The datas will be a useful information for future molecular and genetic studies on sweet osmanthus. Osmanthus fragrans, a woody aromatic plant mainly distributed in China and famous for its beauty and fragrance, is rich in flavonoids. The accumulation of flavonoids is promoted by upregulation of $\mathrm{CHS}, \mathrm{CHI}, \mathrm{F} 3 \mathrm{H}$, and $\mathrm{FLS}$ genes. In the present study, we compared the expression patterns of these genes in 'Dangui' and 'Yingui' petals. The $P A L 1, C H S, F 3 H$, and $F L S$ expressions were higher and $D F R$ and $A N S$ expressions were lower in 'Yingui' than in 'Dangui' petals. The expression profiles reflected the increased synthesis of flavonoids. The $F 3 H$ and 
FLS, which increased 3.9- and 10.9-fold, respectively, in 'Yingui' flowers, are likely the two key genes in flavonoid biosynthesis regulation. The expressions of $D F R$ and $A N S$ were very low compared with those of $C H S, F 3 H$, and $F L S$, which explains the low capability of sweet osmanthus to synthesize anthocyanins. These results corroborate earlier studies on $O$. fragrans, which showed that the content of carotenoids is positively correlated with petal coloration (Han et al. 2013, 2014a). Thus, the anthocyanins are not the main pigments to determine flower color in sweet osmanthus.

Genetic and physiological evidence has shown that JA regulates the WD-repeat/bHLH/MYB complex-mediated anthocyanin accumulation in Arabidopsis thaliana (Qiet al. 2011). JA-inducible MYB14 leads to the accumulation of flavonoids in conifer trees (Bedon et al. 2010). A previous study showed that JA can induce the expression of some transcription factors (such as MYBs), thus promoting the expressions of genes related to the flavonoid biosynthesis pathway and accumulation of flavonoids. The genes $P A L$, $C H S, F 3 H$, and FLS were upregulated, and DFR and ANS were downregulated when the flowers were treated with JA, promoting the accumulation of flavonoids in sweet osmanthus flowers. These results are thus consistent with previous studies.

Salicylic acid can be used as a chemical elicitor to promote the synthesis of total flavonoids in a suspension culture of Andrographis paniculata (Mendhulkar et al. 2013). The expression of the $L c C H I$ gene and the content of total flavonoids in the Lycium chinense berries are upregulated by SA treatment (Guan et al. 2014). In the present study, several upstream genes of the flavonoid biosynthesis pathway were upregulated whereas the expressions of downstream genes remained nearly constant when the flowers were treated with SA, promoting the accumulation of flavonoids in sweet osmanthus flowers. Salicylic acid may activate some transcription factors that regulate the flavonoid biosynthesis pathway.

The exogenous ABA enhances PAL activity and promotes the accumulation of flavonoids in Ginkgo biloba (Hao et al. 2010). Abscisic acid also controls ripening process and regulates the blue berry flavonoid biosynthesis pathway (Zifkin et al. 2012). In the present paper, ABA treatment had no significant effect on the expressions of the genes involved in the flavonoid biosynthesis pathway. These results are thus not consistent with other studies.

Flavonoid and anthocyanin biosynthesis pathways are regulated by MYB transcription factors. The MYBs regulating the flavonol branch of the flavonoid biosynthesis pathway have also been identified in Arabidopsis and grapevine (Mehrtens et al. 2005, Stracke et al. 2007, Czemmel et al. 2009). Our previous research showed that the expression profile of OfMYB1 is not consistent with those of $P A L, C H S$, and $C H I$ suggesting that MYB1 does not regulate these genes and flavonoid biosynthesis pathway (Han et al. 2015). However, the results of the yeast one-hybrid system showed that MYB1 can bind to the promoter of OfPAL and thus regulate its expression (Han et al. 2015). In the present paper, the results demonstrate that the overexpression of MYB1 enhanced the expression of $P A L, P A L 1, C H I$, and FLS, ultimately increasing the accumulation of flavonoids. The increased expression of $P A L$ and $P A L 1$ promoted the biosynthesis of phenylpropanoids including some aromatic compounds, alkaloids, flavonoids, etc. However, the overexpression of MYB1 did not affect other genes related to flavonoid biosynthesis. Taken together, these results suggest that MYB1 and some other transcription factors (e.g., MYBs and bHLH) may co-regulate the expression of phenylpropanoid metabolic pathway-related genes. Future inquiries should aim to discover additional transcription factors in order to elucidate the mechanism of flavonoid biosynthesis.

\section{References}

Altschul, S.F., Gish, W., Miller, W., Myers, E.W., Lipman, D.J.: Basic local alignment search tool. - J. mol. Biol. 215: 403410, 1990.

Bairoch, A., Apweiler, R.: The SWISS-PROT protein sequence data bank and its supplement TrEMBL in 1999. - Nucl. Acids Res. 28: 49-54, 1999.

Baudry, A., Heim, M.A., Dubreucq, B., Caboche, M., Weisshaar, B., Lepiniec, L.: TT2, TT8, and TTG1 synergistically specify the expression of BANYULS and proanthocyanidin biosynthesis in Arabidopsis thaliana. - Plant J. 39: 366-380, 2010.

Bedon, F., Bomal, C., Caron, S., Levasseur, C., Boyle, B., Mansfield, S.D., Gershenzon, J., Grima-Pettenati, J., Séguin, A., MacKay, J.: Subgroup 4 R2R3-MYBs in conifer trees: gene family expansion and contribution to the isoprenoid- and flavonoid-oriented responses. - J. exp. Bot. 61: 3847-3864, 2010.

Bovy, A.G., De Vos, R., Kemper, M., Schijen, E., Pertejo, M.A., Muir, S., Collins, G., Robinson, S., Verhoeyen, M., Hughes, S.: High-flavonol tomatoes resulting from the heterologous expression of the maize transcription factor genes LC and C1. - Plant Cell 14: 2509-2526, 2002.

Broun, P.: Transcriptional control of flavonoid biosynthesis: a complex network of conserved regulators involved in multiple aspects of differentiation in Arabidopsis. - Curr. Opin. Plant Biol. 8: 272-279, 2005.

Czemmel, S., Stracke, R., Weisshaar, B., Cordon, N., Harris, N.N., Walker, A.R., Robinson, S.P., Bogs, J.: The grapevine R2R3-MYB transcription factor VvMYBF1 regulate flavonol synthesis in developing grape berries. - Plant Physiol. 151: 1513-1530, 2009.

Debeaujon, I., Peeters, A.J., Léon-Kloosterziel, K.M., Koornneef, M.: The TRANSPARENT TESTA12 gene of Arabidopsis encodes a multidrug secondary transporter-like protein required for flavonoid sequestration in vacuoles of the seed coat endothelium. - Plant Cell 13: 853-871, 2001.

Espley, R.V., Hellens, R.P., Putterill, J.,Stevenson, D.E.,KuttyAmma, S.,Allan, A.C.: Red colouration in apple fruit is due to the activity of the MYB transcription factor, MdMYB10. - Plant J. 49: 414-427, 2007.

Finn, R.D., Tate, J., Mistry, J., Coggill, P.C., Sammut, S.J., Hotz, H.R., Ceric, G., Forslund, K., Eddy, S.R., Sonnhammer, E.L., Bateman, A.: The Pfam protein families database. - Nucl. Acids Res. 36: D281-D288, 2007.

Guan, C., Song, X., Ji, J., Li, X.Z., Jin, C., Li, J., Wang, G.: Salicylic acid treatment enhances expression of chalcone isomerase gene and accumulation of corresponding flavonoids 
during fruit maturation of Lycium chinense. - Eur. Food Res. Technol. 239: 857-865, 2014.

Gonzalez, A., Zhao, M., Leavitt, J.M., Lloyd, A.M.: Regulation of the anthocyanin biosynthetic pathway by the TTG1/bHLH/ Myb transcriptional complex in Arabidopsis seedlings. - Plant J. 53: 814-827, 2010.

Grabherr, M.G., Haas, B.J., Yassour, M., Levin, J.Z., Thompson, D.A., Amit, I., Adiconis, X., Fan, L., Raychowdhury, R., Zeng, Q., Chen, Z., Mauceli, E., Hacohen, N., Gnirke, A., Rhind, N., Palma, F.D., Birren, B.W., Nusbaum, C., LindbladToh, K., Friedman, N., Regev, A.: Trinity: reconstructing a full-length transcriptome without a genome from RNA-Seq data[J]. - Nat. Biotechnol. 29: 644-652, 2011.

Han, Y.J., Wang, X.H., Chen, W.C., Dong, M.F., Yuan, W.J., Shang, F.D.: Differential expression of carotenoid-related genes determines diversified carotenoid coloration in flower petal of Osmanthus fragrans. - Tree Genet. Genomes 10: 329$338,2014 a$.

Han, S., Wang, C.W., Wang, W.L., Jiang, J.: Mitogen-activated protein kinase 6 controls root growth in Arabidopsis by modulating $\mathrm{Ca}^{2+}$-based $\mathrm{Na}^{+}$flux in root cell under salt stress. J. Plant Physiol. 171: 26-34, 2014b.

Han, Y.J., Chen, W.C., Yang, F.B., Wang, X.H., Dong, M.F., Zhou, P., Shang, F.D.: cDNA-AFLP analysis on 2 Osmanthus fragrans cultivars with different flower colour and molecular characteristics of OfMYB1 gene. - Trees 29: 931-940, 2015.

Han, Y.J., Liu, L.X., Dong, M.F., Yuan, W.J., Shang, F.D.: cDNA cloning of the phytoene synthase (PSY) and expression analysis of PSY and carotenoid cleavage dioxygenase genes in Osmanthus fragrans. - Biologia 68: 258-263, 2013.

Han, Y.J., Wang, H.Y., Wang, X.D., Li, K., Dong, M.F., Li, Y., Zhu, Q., Shang, F.D.: Mechanism of floral scent production in Osmanthus fragrans and the production and regulation of its key floral constituents, $\beta$-ionone and linalool. - Hort. Res. 6: 106, 2019.

Han, Y.J., Wu, M., Cao, L.Y. Shang, F.D.: Characterization of OfWRKY3, a transcription factor that positively regulates the carotenoid cleavage dioxygenase gene OfCCD4 in Osmanthus fragrans. - Plant Mol. Biol. 91: 485-496, 2016.

Hao, G., Du, X., Zhao, F., Ji, H.: Fungal endophytes-induced abscisic acid is required for flavonoid accumulation in suspension cells of Ginkgo biloba. -Biotechnol. Lett. 32: 305314,2010

Harris, M.A., Clark, J., Ireland, A., Foulger, R.: The Gene Ontology (GO) database and informatics resource. - Nucl. Acids Res. 32: D258-D261, 2004.

He, Y.X., Yuan, W.J., Dong, M.F., Han, Y.J., Shang, F.D.: The first genetic map in sweet osmanthus (Osmanthus fragrans Lour.) using specific locus amplified fragment sequencing. -Front. Plant Sci. 8: 1621, 2017.

Hernandez, J.M., Heine, G.F., Irani, N.G., Feller, A., Kim, M.G., Matulnik, T., Chandler, V.L., Grotewold, E.: Different mechanisms participate in the R-dependent activity of the R2R3 MYB transcription factor C1. - J. biol. Chem. 279: 48205-48213, 2004.

Huse, S.M., Huber, J.A., Morrison, H.G., Sogin, M.L., Welch, D.M.: Accuracy and quality of massively parallel DNA pyrosequencing. - Genome Biol. 8: R143, 2007.

Kanehisa, M., Goto, S., Kawashima, S., Okuno, Y., Hattori, M.: The KEGG resource for deciphering the genome. - Nucleic Acids Res. 32: D277-D280, 2004.

Koes, R., Verweij, W., Quattrocchio, F.: Flavonoids: a colorful model for the regulation and evolution of biochemical pathways. - Trends Plant Sci. 10: 236-242, 2005.

Livak, K.J., Schmittgen, T.D.: Analysis of relative gene expression data using real-time quantitative PCR and the 2
(-Delta Delta C(T)) method. - Methods 25: 402-408, 2001.

Love, M.I., Huber, W., Anders, S.: Moderated estimation of fold change and dispersion for RNA-seq data with DESeq2. Genome Biol. 15: 550, 2014.

Margulies, M., Egholm, M., Altman, W.E., Attiya, S., Bader, J.S.: Genome sequencing in microfabricated high-density picolitre reactors. - Nature 437: 376-380, 2005.

Mathews, H., Clendennen, S.K., Caldwell, C.G., Liu, X.L., Connors, K., Matheis, N., Schuster, D.K., Menasco, D.J., Wagoner, W., Lightner, J.: Activation tagging in tomato identifies a transcriptional regulator of anthocyanin biosynthesis, modification, and transport. - Plant Cell 15: 1689-1703, 2003.

Mendhulkar, V.D., Vakil, M.M.A.: Elicitation of flavonoids by salicylic acid and Penicillium expansum in Andrographis paniculata (Burm. f.) Nees. cell culture. - Res. Biotechnol. 2:1-9, 2013 .

Mehrtens, F., Kranz, H., Bednarek, P., Weisshaar, B.: The Arabidopsis transcription factor MYB12 is a flavonol-specific regulator of phenylpropanoid biosynthesis. -J. Plant Physiol. 138: 1083-1096, 2005.

Mondal, S.K., Roy, S.: Genome-wide sequential, evolutionary, organizational and expression analyses of phenylpropanoid biosynthesis associated MYB domain transcription factors in Arabidopsis. - J. Biomol. Struct. Dynamics 36: 1577-1601, 2017.

Morita, Y., Saitoh, M., Hoshino, A., Nitasaka, E., Iida, S.: Isolation of cDNAs for R2R3-MYB, bHLH and WDR transcriptional regulators and identification of $\mathrm{c}$ and ca mutations conferring white flowers in the Japanese morning glory. - Plant Cell Physiol. 47: 457-470, 2006.

Nakamura, N., Masako, F.M., Miyazaki, K., Tanaka, Y.: RNAi suppression of the anthocyanidin synthase gene in Torenia hybrida yields white flowers with higher frequency and better stability than antisense and sense suppression. - Plant Biotechnol. 23: 13-17, 2006.

Nakatsuka, T., Abe, Y., Kakizaki,Y., Yamamura, S., Nishihara, M.: Production of red-flowered plants by genetic engineering of multiple flavonoid biosynthetic genes. - Plant Cell Rep. 26: 1951-1959, 2007.

Nesi, N., Jond, C., Debeaujon, I., Caboche, M., Lepiniec, L.: The Arabidopsis TT2 gene encodes an R2R3 MYB domain protein that acts as a key determinant for proanthocyanidin accumulation in developing seed. - Plant Cell 13: 2099-2114, 2001.

Nishihara, M., Nakatsuka, T., Yamamura, S.: Flavonoid components and flower color change in transgenic tobacco plants by suppression of chalcone isomerase gene. - FEBS Lett. 579: 6074-6078, 2005.

Novaes, E., Drost, D.R., Farmerie, W.G., Pappas, G.J.Jr., Grattapaglia, D., Sederoff, R.R., Kirst, M.: High-throughput gene and SNP discovery in Eucalyptus grandis, an uncharacterized genome. - BMC Genomics 9: 312, 2008.

Peters, D.J., Constabel, C.P.: Molecular analysis of herbivoreinduced condensed tannin synthesis: cloning and expression of dihydroflavonol reductase from trembling aspen (Populus tremuloides). - Plant J. 32: 701-712, 2002.

Qi, T., Xie, D.: The jasmonate-ZIM-domain proteins interact with the WD-Repeat/bHLH/MYB complexes to regulate jasmonate-mediated anthocyanin accumulation and trichome initiation in Arabidopsis thaliana. - Plant Cell 23: 1795-1814, 2011.

Quattrocchio, F., Verweij, W., Kroon, A., Spelt, C., Mol, J., Koes, R.: PH4 of Petunia is an R2R3 MYB protein that activates vacuolar acidification through interactions with basic-helixloop-helix transcription factors of the anthocyanin pathway. 
- Plant Cell 18: 1274-1291, 2006.

Quattrocchio, F., Wing, J., Van der Woude, K., Souer, E., De Vetten, N., Mol, J., Koes, R.: Molecular analysis of the anthocyanin 2 gene of petunia and its role in the evolution of flower colour. - Plant Cell 11: 1433-1444, 1999.

Ramsay, N.A., Glover, B.J.: MYB-bHLH-WD40 protein complex and the evolution of cellular diversity. - Trends Plant Sci. 10: 63-70, 2005.

Schwinn, K., Venail, J., Shang, Y., Mackay, S., Alm, V., Butelli, E., Oyama, R., Bailey, P., Davies, K., Martin, C.: A small family of MYB-regulatory genes controls floral pigmentation intensity and patterning in the genus Antirrhinum. - Plant Cell 18: 831-851, 2006.

Seitz, C., Vitten, M., Steinbach, P., Hartl, S., Hirsche, J., Rhthje, W., Treutter, D., Forkmann, G.: Redirection of anthocyanin synthesis in Osteospermum hybrida by a two-enzyme manipulation strategy. - Phytochemistry 68: 824-833, 2007.

Sonnhammer, E.L.L., Koonin, E.V.: Orthology, paralogy and proposed classification for paralog subtypes. - Trends Genet. 18: 619-620, 2003.

Spelt, C., Quattrocchio, F., Mol, J.N.M., Koes. R.: Anthocyanin1 of Petunia encodes a basic helix-loop-helix protein that directly activates transcription of structural anthocyanin genes. - Plant Cell 12: 1619-1632, 2000.

Stracke, R., Ishihara, H., Huep, G., Barsch, A., Mehrtens, F., Niehaus, K., Weisshaar, B.: Differential regulation of closely related R2R3-MYB transcription factors controls flavonol accumulation in different parts of the Arabidopsis thaliana seedling. - Plant J. 50: 660-677, 2007.

Takos, A.M., Jaffé, F.W., Jacob, S.R., Bogs, J., Robinson, S.P., Walker, A.R.: Light-induced expression of a MYB gene regulates anthocyanin biosynthesis in red apples. - Plant Physiol. 142: 1216-1232, 2006.

Tanaka, Y., Ohmiya, A.: Seeing is believing: engineering anthocyanin and carotenoid biosynthetic pathways. - Curr. Opin. Biotechnol. 19: 190-197, 2008.

Tanaka, Y., Sasaki, N., Ohmiya, A.: Biosynthesis of plant pigments: anthocyanins, betalains and carotenoids. - Plant J. 54: 733-749, 2010.
Tatusov, R.L., Fedorova, N.D., Jackson, J.D., Jacobs, A.R., Kiryutin, B., Koonin, E.V., Krylov, D.M., Mazumder, R., Mekhedov, S.L., Nikolskaya, A.N., Rao, B.S., Smirnov, S., Sverdlov, A.V., Vasudevan, S., Wolf, Y.I., Yin, J.J., Natale, D.A.: The COG database: an updated version includes eukaryotes. - BMC Bioinformatics 4: 41, 2003.

Tsuda, S., Fukui, Y., Nakamra, N., Katsumoto, Y., Sakakibara, K., Fukuchi-Mizutani, M., Ohira, K., Ueyama, Y., Ohkawa, H., Holton, T.A., Kusumi, T., Tanaka, Y.: Flower colour modification of Petunia hybrida commercial varieties by metabolic engineering. - Plant Biotechnol. 21: 377-386, 2004.

Vimolmangkang, S., Han, Y., Wei, G.C., Korban, S.S.: An apple MYB transcription factor, MdMYB3, is involved in regulation of anthocyanin biosynthesis and flower development. - BMC Plant Biol. 13: 176, 2013.

Wang, Z., Gerstein, M., Snyder, M.: RNA-Seq: a revolutionary tool for transcriptomics. - Nat. Rev. Genet. 10: 57-63, 2009.

Wei, W.L., Qi, X.Q., Wang, L.H., Zhang, Y.X., Hua, W., Li, D.H., Zhang, X.R.: Characterization of the sesame (Sesamum indicum L.) global transcriptome using Illumina paired-end sequencing and development of EST-SSR markers. - BMC Genomics 12: 451, 2011.

Winkel-Shirley, B.: Flavonoid biosynthesis. A colourful model for genetics, biochemistry, cell biology, and biotechnology. Plant Physiol. 126: 485-493, 2001.

Xiang, Q.B. Liu, Y.L. An Illustrated Monograph of the Sweet Osmanthus Varieties in China. - Zhejiang Science \& Technology Press, Hangzhou 2007.

Zifkin, M., Jin, A., Ozga, J.A., Zaharia, L.I., Irina, L., Schernthaner, J.P., Gesell, A., Abrams, S.R., Kennedy, J.A., Constabel, C.P.: Gene expression and metabolite profiling of developing highbush blueberry fruit indicates transcriptional regulation of flavonoid metabolism and activation of abscisic acid metabolism. - Plant Physiol. 158: 200-224, 2012.

Zuker, A., Tzfira, T., Ben-Meir, H., Ovadis, M., Shklarman, E., Itzhaki, H., Forkamnn, G., Martens, S., Nata-Sharir, I., Weiss, D., Vainstein, A.: Modification of flower colour and fragrance by antisense suppression of the flavanone 3-hydroxylase gene. - Mol Breed. 9: 33-41, 2002. 